\title{
Paediatric Radiotherapy in Morocco
}

\section{Sahraoui $S^{*}$}

Mohammed VI center for treatment and prevention of cancer, University Hospital of Casablanca, Medical school of Medicine of Casablanca, Morocco

*Corresponding author: Sahraoui S, Mohammed VI center for treatment and prevention of cancer, University Hospital of Casablanca, Medical school of Medicine of Casablanca, Morocco, E-mail: sahraouisouha@gmail. com

Citation: Sahraoui S (2016) Paediatric Radiotherapy in Morocco. J Cancer Sci Clin Oncol 3(2): 204. doi: $10.15744 / 2394-6520.3 .204$

Received Date: June 11, 2016 Accepted Date: September 06, 2016 Published Date: September 08, 2016

\begin{abstract}
In Morocco, through the finding of the Casablanca population-based registry, all cancers are estimated at 35000 new cases par year and $3.2 \%$ are aged less or equal 20 years [1]. In our centre, we treated patient from Casablanca region and the half south of Morocco and we treated children and adults in the same unit. During ten years (2004-2014), we've irradiated 5510 children that represented 4\% of all patients. The most frequent tumour were the CNS tumours (20.7\%) followed by lymphoma (20.7\%) specially Hodgkin disease in $75 \%$ of all lymphoma. The bone sarcoma was noted in $17.9 \%$ and the carcinoma and the epithelial tumours were $16 \%$. We needed anaesthesia for the simulation and the treatment (for all the sessions) in $1 \%$ of all cases. We started using conformational radiation therapy since 2008. The locally stages were noted in $75 \%$ correlated with the delay of diagnosis. All children were treated by the 2 linear accelerators during all the day especially during the morning. The median time to start radiation was more than 1 month in $68 \%$ and $12 \%$ more than 3 months. We used the SIOP recommendations and all cases were discussed during the multidisciplinary paediatric oncology committee of the hospital. More time was necessary for the treatment planification since the CT simulation, dosimetry and given radiation.
\end{abstract}

Keywords: Children; Paediatric radiotherapy; Cancer; Availability and application

\section{Introduction}

Pediatric cancers represent less than $1 \%$ of all cancer. It's usually not inherited, although there are some well-studied syndromes such as neurofibromatosis and Li-Fraumeni, which predisposes to cancer, and some cancers, such as retinoblastoma, that are often heritable. Children' type cancers are very different than for adults. In adults, the most common cancers are, breast, prostate, and lung [2].

Radiotherapy is a part of multimodal therapy concepts in pediatric oncology. Significant increase in survival in pediatric oncology was reported in the last 25 years, and the Conventional RT is frequently associated with severe side effects like Growth and musculoskeletal, endocrine and fertility disorders, neuropsychological and secondary cancers [3]. However, the absolute number of irradiated children is estimated to be quite low and recently new techniques like intensity modulated radiation therapy, protontherapy are used in pediatric oncology and can reduce the late effects.

The aim of this article was to report our activity in pediatric radiotherapy for children and to show future perspectives in Morocco.

\section{Patients and Methods}

A descriptive retrospective study was conducted in Mohammed VI center of treatment of cancer of Casablanca. Were included all children aged less or equal 20 years treated between 2004-2014. Demographic and clinical were evaluated, age, sex, localisation, type of tumor according the cancer's WHO classification and stage. To approach the difficulties to pediatric radiation access we analyse the distance between the treatment facility and the home of the children and the different parts of the planning treatment. The data was collected retrospectively using the medical records. All statistical analyses were performed using the commercially available program SPSS 16.0.

\section{Results}

Between 2004 and 2014, 1510 patients treated were aged less or equal to 20 years in our center. They represented $4 \%$ of all patients. All children received radiation therapy included in multimodal treatment. The sex-ratio male/female was 1.2. 
According the referential structure, 52\% lived in Casablanca region, 12.4\% lived 200-300 km far from our center and $10.3 \% 500$ to $600 \mathrm{~km}$ (Table 1).

\begin{tabular}{|c|c|c|c|}
\hline Regions & $\begin{array}{c}\text { Approximate } \\
\text { Distance to }\end{array}$ & Number & Percent \\
\hline our Center $(\mathbf{K m})$ & - & 785 & 52 \\
\hline Masablanca - Settat & $200-300$ & 138 & 12.4 \\
\hline Daraa - Tafillalet & $500-600$ & 155 & 10.3 \\
\hline Souss - Massa & $450-550$ & 155 & 10.1 \\
\hline Beni Mellal - Khénifra & $200-300$ & 147 & 9.8 \\
\hline Goulmim - Ouadnoun & $600-700$ & 30 & 2 \\
\hline Fes - Meknes & $250-350$ & 28 & 1.9 \\
\hline Rabat - Salé - Kenitra & $100-200$ & 6 & 0.4 \\
\hline Tanger - tétouan & $200-300$ & 6 & 0.4 \\
\hline Laayoune - Sakia Al & $1000-1100$ & 6 & 0.4 \\
\hline Hamra & $600-700$ & 3 & 0.2 \\
\hline Oriental - rif & $1500-1600$ & 2 & 0.1 \\
\hline Dakhla - Oued Eddahab & - & 1510 & 100 \\
\hline Total & & & 610 \\
\hline
\end{tabular}

Table 1: Geographic repartition of children treated at the Mohammed VI Center of Treatment and Prevention of Casablanca06)

The children we treated with adults in the same unit but they received the chemotherapy or surgery in the others pediatric units.

The most frequent tumour were the CNS tumours (20.7\%) followed by lymphoma (20.7\%) specially Hodgkin disease in $75 \%$. The bone sarcoma was noted in $17.9 \%$ and the carcinoma and retinoblastoma in $16.6 \%$ for each one (Table 2). For CNS tumors, the tumor was localized in the posterior cerebral fossa in $44.1 \%$ and in the supratentorial areas in $32.4 \%$. Medulloblastoma was the common tumor of the posterior cerebral fossa in $53 \%$ followed by the ependymoma $30.6 \%$. Glioblastoma was the frequent grade in the astrocytoma tumors with $67.4 \%$ of CNS tumors. In medulloblastoma, the average age was 9 years, and sex - ratio male/ female 1.8 .

\begin{tabular}{|c|c|c|}
\hline Localisation & Number & Percent \\
\hline CNS & 312 & 20,7 \\
\hline Lymphoma & 312 & 20,7 \\
\hline Bone tumors & 270 & 17,9 \\
\hline Retinoblastoma & 250 & 16,6 \\
\hline Kidney (NPB) & 93 & 6,2 \\
\hline Soft Sarcomas & 93 & 6,2 \\
\hline Germ cell tumors & 69 & 4,7 \\
\hline Leukaemia & 38 & 2,6 \\
\hline $\begin{array}{c}\text { Peripheral } \\
\text { nervous system }\end{array}$ & 33 & 2,2 \\
\hline Retinoblastoma & 18 & 1,3 \\
\hline Liver & 10 & 0,7 \\
\hline Others & 3 & 0,2 \\
\hline Total & 1510 & 100 \\
\hline
\end{tabular}

CNS: Centrel nervous system tumors, NPB: nephroblastoma

Table 2: Cancer frequency in the Mohammed VI center of treatment of Cancer

Stages T3-4 were the most frequent with $49 \%$ stage T3 and 17\% stage T4 and 55\% were without metastasis. Only $40 \%$ had complete resection but $70 \%$ had residual tumor more than $1.5 \mathrm{~cm}^{2}$.

After the first medical consultation $2.2 \%$ of children aged less or equal 5 years were cooperating for the treatment. In 93 cases, we brought the children a week before the scanner simulation to familiarize them to technical platform team including manipulators, radiotherapist and to explain the contention with masks if necessary and how to make the sessions radiotherapy. We used aesthesia for the all sessions of radiotherapy in 28 cases. Until 2008 all patient were treated by 2 D radiotherapy with Cobalt 60 machine (Figure 1) and after we used conformational 3D radiation therapy by 2 linear accelerators during all the day especially during the 
morning. All children, had system contention to minimize the shifting (Figures 2 and 3). Five girls had ovarian transposition, 2 for medulloblastomas and 3 for pelvic rhabdomyosarcomas (Figure 4). Regarding the stage of the tumor at the diagnosis, 75\% of patients had a locally advanced stages and the median time to start was more than 1 month in $68 \%$ and $12 \%$ more than 3 months.

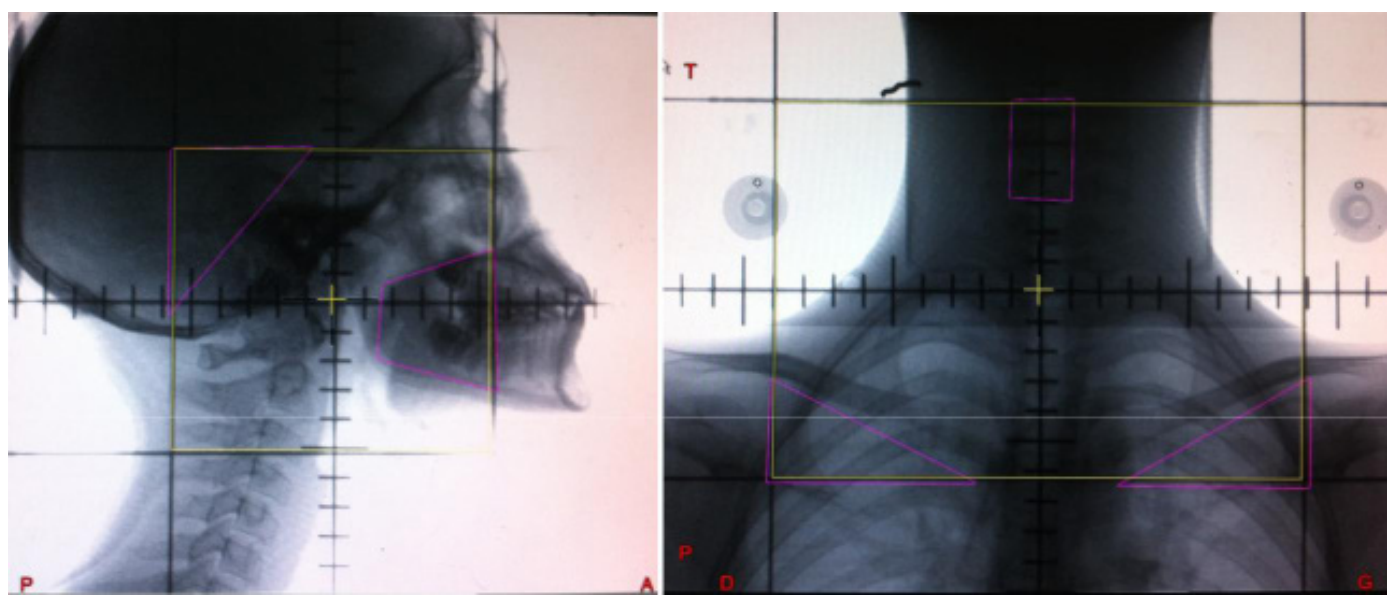

Figure 1: 2D irradiation for a 17 years old boy treated before 2008 for nasopharyngeal carcinoma T3N0M0 first plan 46Gy followed by the boost in the nasopharynx to $70 \mathrm{~Gy}$
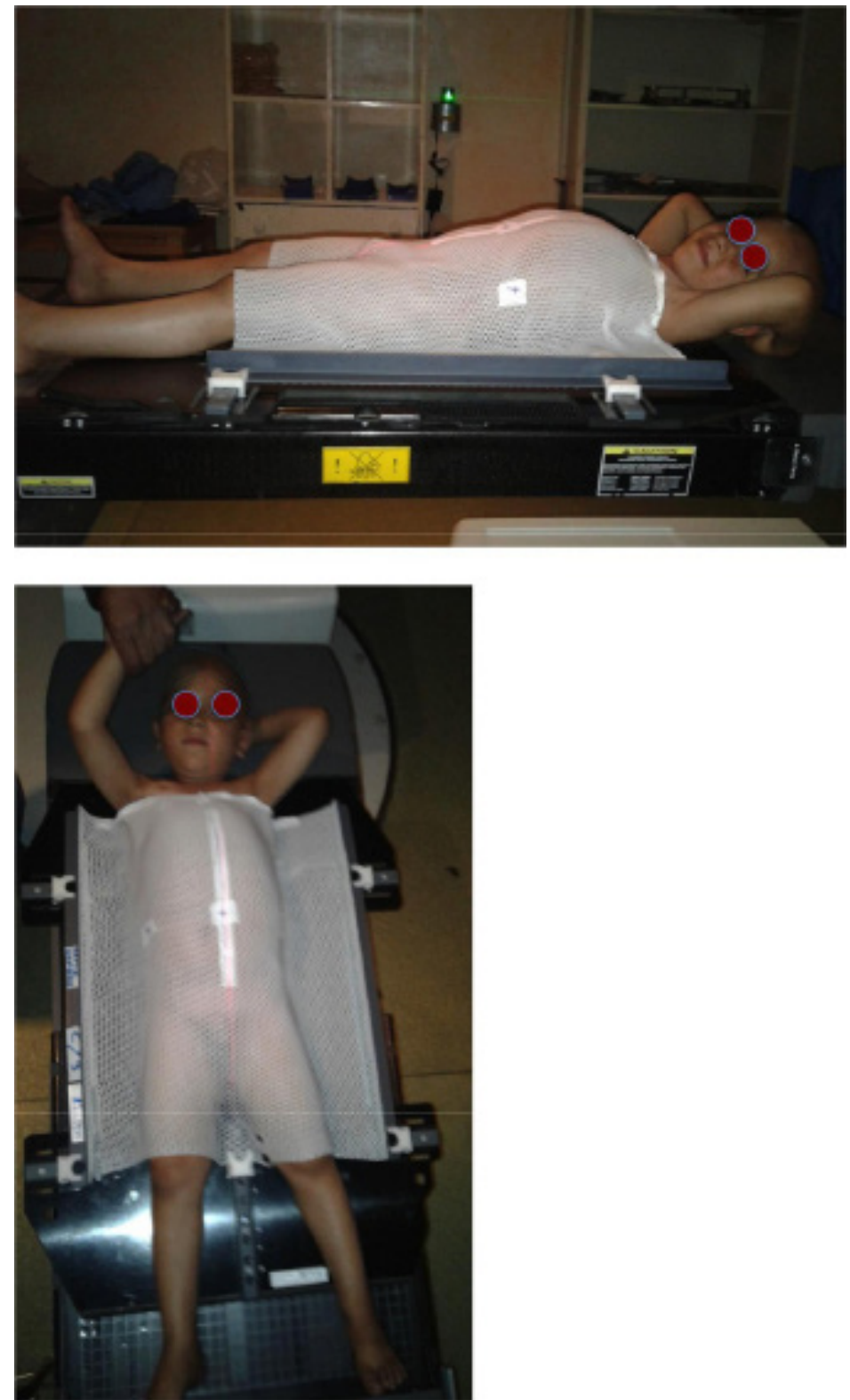

Figures 2 and 3: 6 years old boy with abdominal contention for left nephroblastoma stage III 


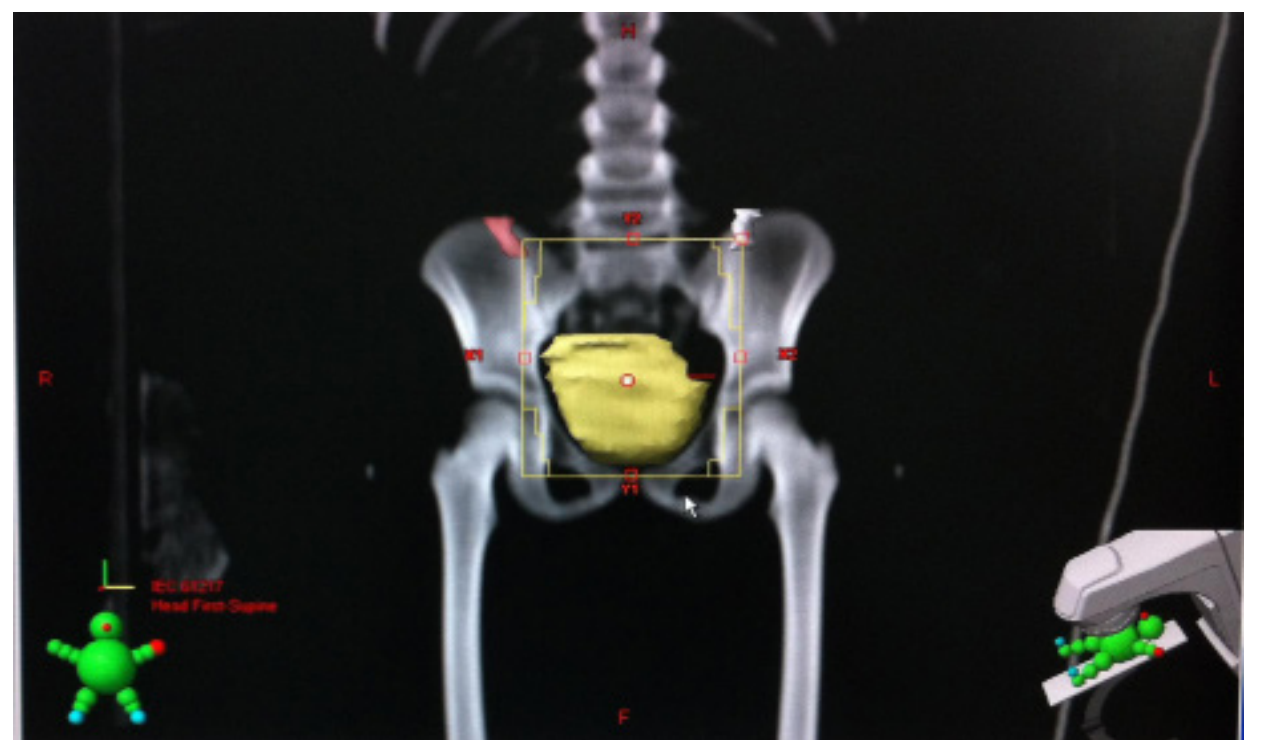

Figure 4: Delineation of the organ at risk left ovary (white), right ovary (red) after transposition and bladder (yellow)

We used the SIOP recommendations and all cases were discussed during the weekly multidisciplinary paediatric oncology committee of the hospital with surgeons, pediatric oncologists, pathologists, radiologists and radiotherapists.

The median time to start radiation was more than 1 month in $68 \%$ and $12 \%$ more than 3 months. In medulloblastoma, the radiation delay after surgery was more than 40 days in $83 \%$ of cases. More time was necessary for the treatment planification since the CT simulation, dosimetry and given radiation.

\section{Discussion}

The Kingdom of Morocco is located in the western north of the African continent and has a total population of 33848242 habitants (Census 2014) with $710850 \mathrm{~km}^{2}$ [4]. The Greater Casablanca is one of the 16 regions composing the Kingdom with a total population 4270750 according to the 2014' census (12.6\% of Moroccan total population) and $37.1 \%$ are younger than 20 witch $24.6 \%$ are boys. The Mohammed VI center is the old radiation department in Morocco built in 1927, followed by the National institute of Oncology in Rabat since 1984. Usually, Casablanca's center treated the half south population of Morocco and Rabat the half north.

Since the creation of Lalla Salma foundation for the treatment and prevention of cancer in 2005, the morocco count 7 new publics centers versus 2. The same observation is noted for private units. Casablanca based-population registry is the first registry in Morocco and these results can be extrapolated to the total Moroccan population [1]. We treated 1510 new children and adolescents (age $<20$ years); the CNS tumors represented 20.7\%, lymphoma in $20.7 \%$ and bone sarcoma in $17.9 \%$. According the registry all cancers are estimated at 35000 new cases per year and 3.2\% are aged less or equal 20 years. The incidence of cancer in patients under 15 years of age in Morocco is estimated to be 1000 new cases per year and the incidence of leukaemia to be 100 new cases diagnosed per year [5].

During 3 years (2005, 2006 and 2007), 530 children were treated; 293 were boys (sex-ratio = 1.2 male/female). The standardised incidence was 14.1 per 100000 habitants. In this pediatric population, the most frequent tumors are CNS with 16\%, followed by leukaemia with $12 \%$ and bone tumors in $10 \%$. Boys are predominant for the majority of the tumors except for nephroblastoma. So we treated more CNS tumors, lymphoma, bone tumors and retinoblastoma than cases registered in the population-based Casablanca registry (Table 3). Overall, cancers in children aged 0-14 years in Rabat represent $2 \%$ of all cancers, and their incidence is twice as high among males than females (15.2 versus 8.2/100,000 children). About $43 \%$ of cancers in childhood are malignant haemopathies (leukaemia: 29\%, lymphoma: $10 \%$ and others: $4 \%$ ), and the other frequent localisations are the brain (18\%), kidney (14\%), and eye (8\%) [6].

Until 2011, we received children from the half south of Morocco and now they are treated in Marrakech. Pediatric cancers remained have a good prognosis than adults, A comprehensive overview and analysis of the SEER pediatric cancer data, providing incidence and survival rates by type and age of diagnosis for the years between 1975 and 2006. It can be considered a big data evaluating the survivals. For all types of pediatric cancers combined, the chances of surviving one's cancer is now about $79 \%$ compared to just 63\% 30 years ago [7]. Bölling T, et al. reported the availability and application of the pediatric radiation in Germany in 2015 [8]. Theirs results demonstrate quite low patient numbers in the different radiotherapy facilities in pediatric radiation oncology. The calculated number of 1.140 to 1.160 children and adolescents treated with radiotherapy each year revealed quite high results in comparison to estimations of 500-600 or 800 patients per year. Some inaccuracy may have been included by the patient number calculation. 


\begin{tabular}{|c|c|c|c|c|}
\hline & \multicolumn{2}{|c|}{ Casablanca registry (1) } & \multicolumn{2}{c|}{ Our study } \\
\hline $\begin{array}{c}\text { Localisation } \\
\text { (ICCC) }\end{array}$ & Number & $\%$ & Percent & $\%$ \\
\hline CNS & 312 & 16 & 85 & 20,7 \\
\hline Lymphoma & 312 & 14.5 & 75 & 20,7 \\
\hline Bone tumors & 270 & 10 & 53 & 17,9 \\
\hline Retinoblastoma & 250 & 9.7 & 51 & 16,6 \\
\hline Kidney (NPB) & 93 & 8.4 & 44 & 6,2 \\
\hline Soft Sarcomas & 93 & 7.8 & 42 & 6,2 \\
\hline Germ cell tumors & 69 & 6.8 & 36 & 4,7 \\
\hline Leukaemia & 38 & 6.1 & 33 & 2,6 \\
\hline $\begin{array}{c}\text { Peripheral } \\
\text { nervous system }\end{array}$ & 33 & 5.5 & 29 & 2,2 \\
\hline Retinoblastoma & 18 & 5.3 & 28 & 1,3 \\
\hline Liver & 10 & 3.1 & 16 & 0,7 \\
\hline Others & 3 & 6.9 & 37 & 0,2 \\
\hline Total & 1510 & 100 & 530 & 100 \\
\hline
\end{tabular}

CNS: Central Nervous system, RTB: retinoblastoma, NPB: nephroblastoma, NHL: Non Hodgkin lymphoma, HL: Hodgkin lymphoma Table 3: Comparison between Pediatric cancer frequency in the Mohammed VI center of treatment and prevention and according the Casablanca base-population registry

Providing an overview about the approaches to reduce the long-term burden of treatment-related complications in survivors of childhood cancer [9]. Especially children with low median of their age at diagnosis are more expose to late effects. Furthermore, it can be assumed that $75 \%$ of the children and adolescents treated for cancer have not finished growing. Due to the special anatomical conditions in these cases, special experiences are needed. The primordial reason for the high difficulty level for pediatric cases is that every tissue in the irradiated volume is potentially at risk for toxicity and the tolerance doses are frequently lower, sometimes much lower, than for the same tissues in adults, making it more frequently challenging to keep the normal tissue doses below tolerance. In addition, modern conformal treatments that avoid parallel-opposed fields may cause more asymmetric growth, pitting the preservation of organ function against cosmesis. Some structures are critical to protect in children but can be largely ignored in adults [2].

It can be assumed that centers with higher patient numbers can rely on a somewhat more fundamental experience in pediatric radiation oncology than those centers with little patient numbers. Many of the centers with high patient numbers had named special contact person for pediatric radiation oncology to further summarize individual experiences [7].

Detailed recommendations regarding pediatric radiation oncology are also not available. As an example, the "Guidelines for Pediatric Cancer Centers" of the "American Academy of Paediatrics" only recommend "a board-certified radiation oncologist trained and experienced in the treatment of infants, children, and adolescents" as well as an "up to date radiation-therapy equipment with facilities for treating pediatric patients" [10].

For optimal Pediatric radiotherapy we need to considered several aspects. Cooperation with a pediatric oncology unit is of fundamental importance due to the coordination function regarding the multimodal therapy and the need for several specialists for pediatric oncology patient care. Considerations lead to be necessary to restrict radiotherapy in pediatric patients to specialized centers to use their broad experiences. On the other hand, a short distance between the treatment facility and the home of the children may be beneficial in social considerations for the children and their families. In our context, 25\% of patients lived more than $300 \mathrm{~km}$ from the center. Especially in palliative treatment situations a short distance between radiotherapy facilities and the home of the children is very important. Further arguments for a short distance could be found financial problems in view of travelling or hosting costs. Actually, since 2011, a new pediatric radiation unit started in Marrakech. For medulloblastoma, we noted only $40 \%$ of complete resection, it can be explained by the gross tumor at diagnosis, or the delay more than 72 hours for the post operative scan. Additionally, in few cases of children aged less than 5 years, we performed aesthesia and that represented only $23 \%$. The option to perform radiotherapy in sedation or anaesthesia is an important quality sign to treat even very young children. The restricted realization of little children leads to this necessary. However, in many cases anaesthesia can be replaced by a consequent psychological care using methods do reduce fear [11].

Delay to start radiation was more than 1 month in $68 \%$ and more than 3 months in $12 \%$. This can be explained by the high number of children in our center compared to the number of patients reported in the Casablanca based-population registry. Limited resources and limited access to care of the families may also explain the delay in treatment. Now we are installing a new machine $3 \mathrm{D}$ conformational radiation therapy with intensity modulated radiation therapy. For this, we'll increase the number of patient's treated per day, children and adults. 
The value of these special techniques like intensity-modulated radiotherapy (IMRT), stereotactic procedures or brachytherapy for pediatric radiation oncology is unclear [12,13]. IMRT enables us to adapt the high dose area to the target volume in a very conformal way. The values of this technique to achieve higher dose increments to the tumor or to reduce potential side effects or its influence on secondary malignancy induction have to be evaluated by the therapy optimizing trials. The use of these special techniques should be restricted to special centers.

The necessity for a discussion of centralization for several radiotherapy indications will also arise by the fact that there will be some radiotherapy facilities within Morocco who will be able to perform radiotherapy with protons in childhood and adolescence in the future. These recommendations allow local radiotherapy facilities that cannot rely on sufficient experiences in those complex therapy cases to perform a near by home radiotherapy with high quality standards. On the other way, specialized treatment facilities can be performed for very complex treatment situations.

\section{Conclusion}

To improve our results, we'll have to reduce the delay of diagnosis, identify a staff with radiotherapist, dosimeters, technicians and unit to treat this population who is special. We noted that we have children coming from others cities and in some time more than $200 \mathrm{~km}$ so far. Delay to start radiation therapy for children was longer this may decrease the chance of cure. Now we are installing a new machine with intensity modulated radiation therapy. For this we'll dedicate a time section for children or a new machine in the future. In special treatment cases a centralization of radiotherapy in childhood and adolescence.

\section{References}

1. Data presented by the Casablanca based - population registry 2005, 2006 and 2007 (2012).

2. Olch AJ (2010) Overview of Childhood Cancer. In Pediatric Radiotherapy Planning and Treatment. Taylor Francis Group LLC.

3. Demoor-Goldschmidt C, Bernier V (2015) Towards an improvement of the quality of life after radiotherapy in children. Bull Cancer 102: 674-83.

4. Census 2014, High commission for planning.

5. Hessissen L, Madani A (2012) Pediatric Oncology in Morocco: Achievements and Challenges. J Pediatr Hematol Oncol 34: S21-2.

6. Tazi MA, Er-Raki A, Benjaafar N (2013) Cancer incidence in Rabat, Morocco: 2006-2008. Ecancermedicalscience 7: 338.

7. SEER Cancer Statistics Review, 1975-2006.

8. Bölling T, Ernst I, Könemann S, Willich N (2008) Pediatric Radiation Oncology in Germany: a study of availability and application. Klin Pädiatr 220: 178-82.

9. Armenian SH, Leontien DO, Kremer C, Sklar S (2015) ASCO educational Book.

10. Corrigan JJ, Feig SA, American Academy of Pediatrics (2004) Guidelines for pediatric cancer centers. Pediatrics 133: 1833-5.

11. Schreiber-Gollwitzer B, Di Gallo A, Maier S (2007) Position paper by the Gesellschaft für Pädiatrische Onkologie und Hämatologie (GPOH) and the Deutsche Leukämie-Forschungshilfe (DLFH) on psycho-social care for pediatric oncology and haematology patients in hospital. Klin Pädiatr 219: 368-71.

12. Combs S, Behnisch W, Kulozik AE, Huber PE, Debus J, et al. (2007) Intensity modulated radiotherapy (IMRT) and fractionated stereotactic radiotherapy (FSRT) for children with head-and-neck rhabdomyosarcoma. BMC Cancer 7: 177.

13. Paulino AC, Ferenci MS, Chiang KY, Nowlan AW, Marcus RB Jr (2006) Comparison of conventional to intensity modulated radiation therapy for abdominal neuroblastoma. Pediatr Blood Cancer 46: 739-44.

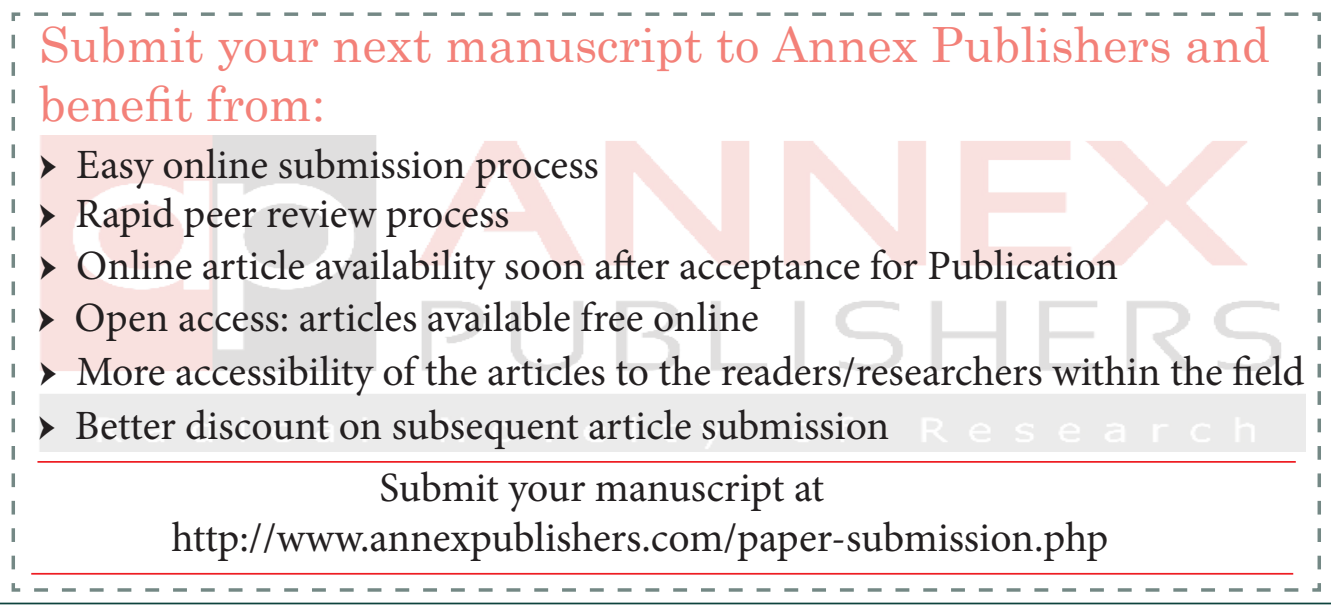

